\title{
Expert-novice differences in SMR activity during dart throwing
}

\author{
Ming-Yang Cheng ${ }^{\mathrm{a}}$, Chiao-Ling Hung ${ }^{\mathrm{b}}$, Chung-Ju Huang ${ }^{\mathrm{c}}$, Yu-Kai Chang ${ }^{\mathrm{d}}$, Li-Chuan Lo $^{\mathrm{e}}$, \\ Cheng Shen ${ }^{\mathrm{b}}$, Tsung-Min Hung ${ }^{\mathrm{b}, *}$ \\ a Cluster of Excellence Cognitive Interaction Technology (CITEC), Bielefeld University, Inspiration 1, 33619 Bielefeld, Germany \\ ${ }^{b}$ Department of Physical Education, National Taiwan Normal University, No. 162, Sec. 1, Heping E. Rd., Da'an Dist., Taipei City 106, Taiwan, ROC \\ ${ }^{\mathrm{c}}$ Graduate Institute of Sport Pedagogy, University of Taipei, No. 101, Section 2, Zhongcheng Rd., Shilin District, Taipei City 111 , Taiwan, ROC \\ ${ }^{\mathrm{d}}$ Graduate Institute of Athletics and Coaching Science, National Taiwan Sport University, No. 250, Wenhua 1st Rd., Guishan Township, Taoyuan County \\ 333, Taiwan, $R O C$ \\ e Department of Kinesiology University of Maryland, College Park, MD 20742, USA
}

\section{A R T I C L E I N F O}

\section{Article history:}

Received 22 August 2014

Received in revised form 28 July 2015

Accepted 6 August 2015

Available online 12 August 2015

\section{Keywords:}

Precision sports

EEG

Sensorimotor rhythm

Efficiency

Attention

\begin{abstract}
A B S T R A C T
Previous evidence suggests that augmented sensorimotor rhythm (SMR) activity is related to the superior regulation of processing cognitive-motor information in motor performance. However, no published studies have examined the relationship between SMR and performance in precision sports; thus, this study examined the relationship between SMR activity and the level of skilled performance in tasks requiring high levels of attention (e.g., dart throwing). We hypothesized that skilled performance would be associated with higher SMR activity. Fourteen dart-throwing experts and eleven novices were recruited. Participants were requested to perform 60 dart throws while EEG was recorded. The 2(Group: Expert, Novice) $\times 2$ (Time window: $-2000 \mathrm{~ms}$ to $-1000 \mathrm{~ms},-1000 \mathrm{~ms}$ to $0 \mathrm{~ms}$ ) ANOVA showed that the dart-throwing experts maintained a relatively higher SMR power than the novices before dart release. These results suggest that SMR might reflect the adaptive regulation of cognitive-motor processing during the preparatory period.
\end{abstract}

(c) 2015 Elsevier B.V. All rights reserved.

\section{Introduction}

Decreased activation in the sensorimotor cortex may represent adaptive information processing in motor execution. It is generally accepted that skillful performers execute movement with lower conscious processing during execution as reflected by less cognitive involvement, thus resulting in less variation in the routine of movement execution. For example, expert golfers showed a relatively lower overall cortical activation than that of novices (Milton, Solodkin, Hluštík, \& Small, 2007). In the same vein, pianists exhibited lower activation compared to novices in a complex motor sequence task in an fMRI study (Meister et al., 2005). In addition to these cross-sectional studies, learning studies have also shown that lower activity at C3 and C4 are observed in marksmen after practice (Kelly \& Garavan, 2005; Petersen, van Mier, Fiez, \& Raichle, 1998). All these studies point to either structural or functional changes in

\footnotetext{
* Corresponding author.

E-mail addresses: ming-yang.cheng@uni-bielefeld.de (M.-Y. Cheng), musehung@gmail.com (C.-L. Hung), crhwang@tpec.edu.tw (C.-J. Huang), yukaichangnew@gmail.com (Y.-K. Chang), llo@umd.edu (L.-C. Lo), supertlor@gmail.com (C. Shen), ernesthungkimo@yahoo.com.tw (T.-M. Hung).
}

the motor cortex after long-term practice (Dayan \& Cohen, 2011). Lower activation in the sensorimotor cortex may be associated with lesser cognitive involvement during the execution of movement, as a result of being related to decreased control in monitoring the processing of motor performance, which leads to more adaptive motor performance.

Sensorimotor rhythm (SMR), the $12-15 \mathrm{~Hz}$ oscillation in the sensorimotor cortex, is an EEG candidate for the investigation of differences in sensorimotor activation in skilled visuo-motor activities. SMR is one of the attention-related indicators (Egner \& Gruzelier, 2001, 2004; Hoedlmoser et al., 2008) that is negatively related to the activity of the sensorimotor cortex (Mann, Sterman, \& Kaiser, 1996). The transmission of somatosensory information through the ventrobasal thalamus was shown to be inhibited during SMR activity in animal studies (Howe \& Sterman, 1973). In other words, SMR power is positively related to the inhibition of sensorimotor cortex activity. SMR neurofeedback training (SMR NFT) studies have shown that individuals can increase SMR power to reduce somatosensory interference during the information processing phase to maintain perception and attention (Vernon et al., 2003). Moreover, augmented SMR power by NFT has been related to motor inhibition and the facilitation of relaxed attention focusing, increases in working memory capacity, better motor preparation 
(Gruzelier, Egner, \& Vernon, 2006), and calming effects on mood (Gruzelier, 2014a). Therefore, higher SMR power might represent the suppression of irrelevant information input from activities in the sensorimotor cortex, which facilitates the formation of relaxed focus (Gruzelier, 2014a) and results in more adaptive preparation for action (Gruzelier et al., 2006).

However, investigations of SMR activity and motor performance are scarce. Gruzelier, Inoue, Smart, Steed, \& Steffert (2010) used virtual reality to increase SMR activity in a monologue dance. The results demonstrated that greater SMR activity was related to better overall monologue dancing performance, but they did not record SMR activity during the actual execution of the skill. The present study attempted to extend our understanding of SMR and the relevance of motor performance by comparing SMR activity between dart-throwing experts and novices during the preparation period. It was hypothesized that experts would exhibit higher SMR power than those of novices prior to skill execution.

\section{Method and design}

\subsection{Participants}

Fourteen dart-throwing experts (mean age $=41.86, \mathrm{SD}=13.79$ years) and eleven novice dart-throwing athletes ( mean age $=22.04$, $\mathrm{SD}=2.09$ years) were recruited. The experts had practiced dart throwing for 13.93 years $(S D=10.02$ ). In order to familiarize participants to the dart-throwing task, novices were allowed to practice before actual testing and EEG recording were conducted. All of the participants were right-handed and did not have any nervous system disease. All participants provided their informed consent and were approved by the institutional review board of the University of Taipei for the protection of human subjects. The participants had the right to withdraw from the study at any time.

\subsection{Procedures}

Participants were asked to refrain from having drinks containing alcohol or caffeine for at least $24 \mathrm{~h}$ prior to their arrival at the laboratory.

\subsection{Task}

All participants were asked to perform dart-throwing tasks. The distance from the front of the dartboard to the throwing line was $2.37 \mathrm{~m}$, and the distance from the floor to the center of the bull'seye was $1.74 \mathrm{~m}$ (consistent with international dart rules). The score was calculated based on the actual scores on the dartboard; the red region of the bull's eye was determined to be 11 , and the outermost circle and a target-miss were calculated as 0 . The dartthrowing task consisted of 60 self-paced dart-throwing trials in 6 separate recording blocks. Participants were asked to commence dart throwing when ready and were instructed to throw as accurately as possible. For the purpose of analyzing the SMR before the onset of the dart-throwing movement, a steady interval of at least $2 \mathrm{~s}$ before the EMG trigger was needed for each trial. Therefore, participants were asked to first hold the dart in front of the chest for $2 \mathrm{~s}$ and use their wrist and elbow for aiming and shooting, with minimum body sway. The standardized instructions were given to each participant in order to reduce confounding effects on EEG and EMG.

\subsection{Electrophysiological recording}

A stretchable Lycra electrode cap (Quick-cap, Neuroscan, Charlotte, NC, USA) was fitted to participants to record their EEG signals from 12 scalp electrodes (i.e., Fz, F3, F4, C3, C4, T3, T4, Pz, P3,
P4, 01 and 02) based on the international 10-20 system (Jasper, 1958). The reference was located at the mastoids to obtain an earaveraged reference offline, and the ground electrode was located at FPz. To monitor blinking and eye movements, vertical and horizontal electrooculograms (VEOG and HEOG, respectively) were recorded with bipolar configurations that were located superior and inferior to the right eye and on the left and right orbital canthi. EEG and EOG signals were sampled at $500 \mathrm{~Hz}$ and amplified 5000 times using Neuroscan Synamps and NeuroScan 4.3 software (Neuroscan, Charlotte, NC, USA), with a band-pass filter setting from 1 to $100 \mathrm{~Hz}$ and a notch filter of $60 \mathrm{~Hz}$. Furthermore, electromyograms (EMGs) were utilized in order to obtain the timing of the onset of the dart-throwing movement. A pair of $\mathrm{Ag} / \mathrm{AgCl}$ electrodes was attached to the flexor digitorum superficialis of right hand: one was located at the one-third position from the wrist and the other was located at the one-third position from the elbow, with bipolar recording used to detect the timing of release upon throwing. The impedance at each electrode was kept under $10 \mathrm{k} \Omega$. In addition, an event marker, activated by pressing a function key, was utilized by the experimenter when the dart-throwing movement was initiated. The event markers were used in subsequent analysis with the EMG signals in order to correctly identify the EMG peaks caused by the initiation of the dart-throwing movement, not a false alarm. After electrode registration, the participants participated in a 10-min warm-up before commencing the formal test. The whole experiment lasted approximately $2 \mathrm{~h}$.

\subsection{Data reduction}

EEG data reduction was conducted offline using Neuroscan Edition 4.5 software (Neuroscan, Charlotte, NC, USA). A band pass of $1-30 \mathrm{~Hz}$ with $12 \mathrm{db} /$ oct FIR filter was applied to the EEG and EOG channels. EOG correction (Semlitsch, Anderer, Schuster, \& Presslich, 1986) was performed on the continuous EEG data to eliminate artifact signals due to blinking. To investigate brain activity prior to the initiation of the dart-throwing movement with a common data structure across trials and participants, EEG data were sampled starting at $2000 \mathrm{~ms}$ prior to the dart release. The timing of the initiation of the dart-throwing movement was determined using $20 \%$ of the EMG amplitude above baseline. Artifact detection as well as eye inspection by the experimenter were performed to screen for unusable trials. Artifacts were defined by amplitudes exceeding $\pm 100 \mu \mathrm{V}$ from baseline (Männel \& Friederici, 2008). The numbers of artifact-free trials for the $-2 \mathrm{~s}$ and $-1 \mathrm{~s}$ epochs before dart throwing were $42(S D=11.17)$ and $33(S D=16.34)$ for dart-throwing experts and $40(\mathrm{SD}=1.91)$ and $40(\mathrm{SD}=1.91)$ for novice dart-throwers, respectively, and were not significantly different $\left(t(23)=.681, p=.601, \eta^{2}=.012\right)$ in a group comparison. After artifact-free EEG data were acquired, Fast Fourier Transforms (FFT) were calculated on $50 \%$ overlapped, 256 sample Hanning windows for all artifact-free segments to transform the C3 and C4 into spectral power $\left(\mu V^{2}\right)$. To examine the sensorimotor activity, the mean power of $\mathrm{C} 3$ and $\mathrm{C} 4$ in the frequency band of $12-15 \mathrm{~Hz}$ was defined to be SMR (Babiloni et al., 2008).

\subsection{Data analysis}

For behavioral data, we compared the dart-throwing scores between two groups with an independent $t$ test.

The SMR power was subjected to a 2 (Group: Expert, Novice) $\times 2$ (Time Window: $-2000 \mathrm{~ms}$ to $-1000 \mathrm{~ms}(\mathrm{~T} 1),-1000 \mathrm{~ms}$ to $0 \mathrm{~ms}$ (T2)) analysis of variance (ANOVA), with repeated measures on the Time Window factor. The Greenhouse-Geisser procedure was employed to correct the degrees of freedom when the sphericity assumption was violated. Simple main effect tests were employed when the interaction effect was significant. The alpha 
Table 1

The SMR power between two groups among T1 $(-2000 \mathrm{~ms} \sim-1000 \mathrm{~ms})$ and T2 $(-1000 \mathrm{~ms} \sim 0 \mathrm{~ms})$ in dart throwing performance.

\begin{tabular}{lll}
\hline & T1 & T2 \\
\hline Expert Group & $1.335(0.542)$ & $1.310(0.654)$ \\
Novice Group & $0.374(0.410)$ & $0.354(0.351)$
\end{tabular}

Unit: $\log \mu \mathrm{V}^{2}$.

level was set at $p<.05$, and the eta square $\left(\mathrm{Eta}^{2}\right)$ was utilized for the estimate of effect size.

\section{Results}

\subsection{Between group comparisons of the performance of dart throwing}

An independent $t$ test on the dart-throwing scores showed a significant group effect $\left(t(27)=8.948, p=.001, \mathrm{Eta}^{2}=.748\right)$. As expected, the experts $(M=9.28, \mathrm{SD}=0.68)$ performed better than the novices $(M=6.44, \mathrm{SD}=1.01)$.

\subsection{Between-group comparisons of SMR power}

The SMR power for the expert group for T1 and T2 was 1.335 $\left(\log \mu \mathrm{V}^{2}\right)(\mathrm{SD}=0.542)$ and $1.310\left(\log \mu \mathrm{V}^{2}\right)(\mathrm{SD}=0.654)$, respectively. For the novice group, the SMR power for T1 and T2 was $0.374 \mu \mathrm{V}^{2}(\mathrm{SD}=0.410)$ and $0.354\left(\log \mu \mathrm{V}^{2}\right)(\mathrm{SD}=0.351)$, respectively. Two-way ANOVA did not show significance for neither interaction $\left(F(1,23)=0.005, p=.942, \mathrm{Eta}^{2}=.000\right)$ nor a main effect for the Time factor $\left(F(1,23)=1.030, p=.321, \mathrm{Eta}^{2}=.043\right)$. However, the main effect of Group was significant $(F(1,23)=21.699, p=.001$, $\left.\mathrm{Eta}^{2}=.485\right)$. An examination of the mean revealed that the experts exhibited a higher SMR power $(M=1.322, \mathrm{SD}=0.137)$ than the novices $(M=0.360, \mathrm{SD}=0.155)$ prior to dart throwing $(t(23)=4.658$, $p=.001)$ (Table 1$)$

\subsection{Control analysis}

\subsubsection{Regional specificity}

In this study, the SMR power (a mean power of $12-15 \mathrm{~Hz}$ in C3 and (4) of the experts was significantly higher than that of the novices during the $2 \mathrm{~s}$ prior to dart throwing. It is unknown whether the greater $12-15 \mathrm{~Hz}$ EEG power was limited to the sensorimotor cortex and the somatosensory integration regions (parietal lobe) (Fogassi \& Luppino, 2005) or if there was fluctuation over the other less relevant regions, such as the occipital cortex and frontal cortex. Accordingly, we expected that a relatively elevated $12-15 \mathrm{~Hz}$ power at the sensorimotor and parietal regions compared to the frontal and occipital regions would be found in the experts. Moreover, based on the cortex efficiency hypothesis (Milton et al., 2007), experts exhibited a generally higher $12-15 \mathrm{~Hz}$ power than the novices, regardless of the regions being examined. Due to insignificant findings regarding the time effect, the SMR results from the two time windows were pooled together. Thus, a 2(Group: Expert, Novice $) \times 4$ (Region: Frontal, Central, Parietal, Occipital) two-way ANOVA mixed design was applied to test the differences in all of the regions. However, in computing the power variation among these regions, establishing a normalized power comparison was needed. Therefore, relative power (\%), defined as the ratio of the mean power of $12-15 \mathrm{~Hz}$ divided by the mean power of $1-30 \mathrm{~Hz}$ corresponding to a given electrode, was employed, which is a common method to investigate whether a certain frequency band is prominent within the designated background activity to establish a normalized comparison among the regions (Niemarkt et al., 2011). The frontal region was represented by $\mathrm{Fz}$, the parietal region used
Table 2

The mean relative power of $12-15 \mathrm{~Hz}$ at the frontal, central, parietal, and occipital regions for the expert and novice groups before dart throwing.

\begin{tabular}{lllll}
\hline & Frontal & Central & Parietal & Occipital \\
\hline Expert group & $0.994(0.185)$ & $1.039(0.181)$ & $1.054(0.195)$ & $1.013(0.062)$ \\
Novice group & $0.709(0.144)$ & $0.842(0.144)$ & $0.851(0.129)$ & $1.001(0.037)$ \\
\hline
\end{tabular}

Unit: \%.

$\mathrm{Pz}$, and the occipital region applied the mean relative power at $\mathrm{O} 1$ and $\mathrm{O} 2$.

It was hypothesized that the relative power of $12-15 \mathrm{~Hz}$ in the sensorimotor and parietal regions would be higher than that of the frontal and occipital regions in experts and that the experts would have a higher $12-15 \mathrm{~Hz}$ power overall than the novices. We found a significant main effect of the Group factor $(F(1$, $\left.23)=12.066, p=.002, \mathrm{Eta}^{2}=.344\right)$, in which the expert group exhibited a higher relative power with a $12-15 \mathrm{~Hz}$ frequency band $(M=1.025, \mathrm{SD}=0.142)$ than that of the novice group $(M=0.855$, $\mathrm{SD}=0.087)(t(23)=3.474, p=.002)$. The results also showed that the effect of the Group $\times$ Region interaction was significant $(F(3$, $69)=10.912, p=.000, \mathrm{Eta}^{2}=.322$ ); subsequent simple main effect analysis revealed that expert group showed a significantly higher relative power of $12-15 \mathrm{~Hz}$ at the frontal $(t(23)=4.563, p=.001)$, central $(t(23)=2.946, p=.007)$, and parietal regions $(t(23)=2.863$, $p=.009$ ) compared to the novice group. In addition, we observed that the novice group exhibited a significantly different relative power within these regions $\left(F(3,30)=25.843, p=.001, \mathrm{Eta}^{2}=.721\right)$. A significantly higher relative power was observed in the occipital, central, and parietal regions relative to that of the frontal region $(t(10)=10.655, p=.001 ; t(10)=4.610, p=.001 ; t(10)=4.989$, $p=.001)$. All the means are presented in Table 2 .

\subsubsection{Frequency specificity}

Although the above analysis demonstrated a distinct $12-15 \mathrm{~Hz}$ power in the sensorimotor region, it is not clear whether the expert-novice difference was only observed on the 12-15 frequency band instead of other EEG markers. Alpha $(8-12 \mathrm{~Hz})$ is associated with sensory information processing over the sensorimotor area (Pfurtscheller \& Lopes Da Silva, 1999). Moreover, the higher alpha power prior to skill execution also predicted a more adaptive golf putting performance in a previous study, which could be interpreted as a sign of fine cognitive-motor performance (Babiloni et al., 2008). In addition, theta $(4-7 \mathrm{~Hz})$ and beta $1(15-18 \mathrm{~Hz})$, the other two neighboring frequency bands, were also included in the examination of the frequency specificity of the present study. Theta, alpha, and beta power (a mean power of $4-7,8-12$ and $15-18 \mathrm{~Hz}$, in C3 and C4) were separately subjected to 2(Group: Expert, Novice) $\times 2$ (Time Window: $-2000 \mathrm{~ms}$ to $-1000 \mathrm{~ms},-1000 \mathrm{~ms}$ to $0 \mathrm{~ms}$ ) analyses of variance (ANOVAs). The results showed that there was no difference in alpha power before dart throwing between experts $(M=1.122, \mathrm{SD}=0.121)$ and novices $(M=0.709, \mathrm{SD}=0.137)\left(F(1,23)=0.149, p=.703, \mathrm{Eta}^{2}=.006\right)$. Neither an interaction effect $\left(F(1,23)=0.012, p=.914, \mathrm{Eta}^{2}=.001\right)$ nor a main effect of the Time factor $(F(1,23)=0.082, p=.380$, $\left.\mathrm{Eta}^{2}=.034\right)$ were observed in our analysis. As for theta power, we found no difference prior to dart throwing between the expert $(M=1.228, \mathrm{SD}=0.084)$ and novice groups $(M=1.235, \mathrm{SD}=0.094)$ $\left(F(1,23)=0.003, p=.956, \mathrm{Eta}^{2}=.003\right)$. Furthermore, the analysis showed that neither the interaction $\operatorname{effect}(F(1,23)=0.156, p=.696$, $\left.\mathrm{Eta}^{2}=.007\right)$ nor the main effect of the Time factor $(F(1,23)=0.001$, $\left.p=.978, \mathrm{Eta}^{2}=.000\right)$ were significant. However, the expert group showed a higher beta1 power $(M=1.235, \mathrm{SD}=0.126)$ than that of the novice group $(M=-0.147, \mathrm{SD}=0.142)$ before dart throwing $(F(1$, $\left.23)=52.818, p=.001, \mathrm{Eta}^{2}=.697\right)$. Neither the interaction effect $\left(F(1,23)=0.318, p=.578, \mathrm{Eta}^{2}=.014\right)$ nor the main effect of the Time 
Table 3

The mean power of theta, alpha and beta 1 at the $\mathrm{C} 3$ and $\mathrm{C} 4$ sites for the expert and novice groups before dart throwing.

\begin{tabular}{llll}
\hline & Theta & Alpha & Beta1 \\
\hline Expert group & $1.228(0.084)$ & $1.122(0.121)$ & $1.235(0.126)$ \\
Novice group & $1.235(0.094)$ & $0.709(0.137)$ & $-0.147(0.142)$
\end{tabular}

Unit: $\log \mu \mathrm{V}^{2}$.

Table 4

The SMR power for the two groups in the resting condition and dart throwing condition resting condition dart throwing condition.

\begin{tabular}{llllll}
\hline & \multicolumn{2}{l}{ Resting condition } & & \multicolumn{2}{l}{ Dart throwing condition } \\
\cline { 2 - 3 } \cline { 5 - 6 } & C3 & C4 & & C3 & C4 \\
\hline Expert group & $1.933(0.531)$ & $1.850(0.509)$ & & $1.357(0.545)$ & $1.346(0.661)$ \\
Novice group & $-0.003(0.476)$ & $-0.174(0.493)$ & & $0.381(0.399)$ & $0.342(0.356)$
\end{tabular}

Unit: $\log \mu V^{2}$.

factor $\left(F(1,23)=2.028, p=.168, \operatorname{Eta}^{2}=.081\right)$ for beta1 power were observed. The means for this analysis are presented in Table 3.

\subsubsection{Task specificity}

In order to determine whether the higher SMR power was taskspecific in dart throwing, we extracted the SMR power from the resting condition in which the participants were instructed to stand up in the same position used when dart throwing and to keep their eyes open, staring at the bull's eye for two minutes. The continuous EEG data were segmented by $2 \mathrm{~s}$ each to obtain the mean SMR power in the resting condition. A 2(Group: Expert, Novice $) \times 2$ (Condition: Resting, Dart Throwing $) \times 2$ (Electrode: C3, C4) three-way ANOVA with Condition and Electrode as the repeated measures was employed. The three-way ANOVA revealed a 2 (Group) $\times 2$ (Condition) interaction effect $(F(1,23)=11.997$, $\left.p=.002, \mathrm{Eta}^{2}=.343\right)$. The simple main effect was significant for the resting condition between the two groups $(F(1,23)=97.008$, $\left.p=.001, \mathrm{Eta}^{2}=.808\right)$, in which the expert group showed higher SMR power than the novice group in the Resting condition. As expected, we also found a significant simple main effect of the Dart Throwing condition $\left(F(1,23)=23.306, p=.001, \mathrm{Eta}^{2}=.503\right)$, in which the expert group exhibited a higher SMR power than the novice group before dart throwing. In addition, we revealed a significant simple main effect within conditions for the expert group $\left(F(1,13)=6.946, p=.021, \mathrm{Eta}^{2}=.348\right)$ and the novice group $\left(F(1,10)=5.682, p=.038, \mathrm{Eta}^{2}=.362\right)$, in which the expert group exhibited lower SMR power in the Dart Throwing condition than in the Resting condition, while the novice group showed higher SMR power in the Dart Throwing condition than in the Resting condition. The group main effect revealed a significant effect $(F(1$, 23) $\left.=105.279, p=.001, \mathrm{Eta}^{2}=.821\right)$ in that the expert group exhibited a generally higher SMR power $(M=1.621, \mathrm{SD}=0.408)$ than the novice group $(M=0.137, \mathrm{SD}=0.283)$, regardless of the condition (see Table 4).

\subsubsection{The EMG prior to movement was higher in the expert group than in the novice group}

We also compared the mean power frequency (MPF) (Vance, Wulf, Töllner, McNevin, \& Mercer, 2004) between the two groups to investigate whether these two groups showed different EMG patterns prior to dart throwing. We computed the EMG signal two seconds before dart throwing and filtered it into $1-100 \mathrm{~Hz}$ frequency bands. Then, we performed an independent $t$ test to examine the means. The results showed that the mean power of EMG was $2.849(\mathrm{SD}=1.276)$ and $0.573(\mathrm{SD}=0.306)$ in the expert and novice groups, respectively. The expert group revealed a sig- nificantly higher EMG power than the novice group $(t(23)=5.754$, $p=.001)$.

\subsubsection{The ocular activity showed no difference for both groups during the preparation period}

In order to minimize the confounding effect of ocular activity between the two groups during the preparation period in dartthrowing task, we further compared the difference in fixation between the novice and expert groups. As previous studies have suggested, the common structure of fixation was defined as a stable gazing position that is steadily maintained for at least $100 \mathrm{~ms}$ (Manor \& Gordon, 2003). The first step to extract the gazing position was to segment the EOG signal with time windows of $100 \mathrm{~ms}$ each for 60 dart-throwing trials. Second, these segmented epochs underwent an artifact rejection process, as they were required to be within $\pm 100 \mu \mathrm{V}$ of baseline to be validated as a stable gazing position. Third, the number of remaining gazing position trials was counted and averaged for every participant. The average number of trials represented the number of fixation. Finally, a $t$ test was performed to compare the number of fixations between the two groups. We hypothesized that both groups shared a similar number of fixations in the preparation period. Results showed that the mean number of fixations in the expert and novice groups was $58.768(\mathrm{SD}=6.421$ ) and 59.636 ( $\mathrm{SD}=1.206)$, respectively. We found no significant differences between these two groups in the $t$ test $(t(23)=0.441, p=.664)$.

\subsubsection{No contralateral effect was observed on the SMR power within the $\mathrm{C} 3$ and $\mathrm{C} 4$ sites}

It is also arguable whether the activity of SMR power showed a contralateral effect before dart throwing in this study. Therefore, this additional analysis was designed to investigate the difference between the SMR power at the C3 and C4 sites for both groups. We extracted the power of $12-15 \mathrm{~Hz}$ from the $\mathrm{C} 3$ and $\mathrm{C} 4$ sites with a two-second segment before dart throwing. Then, we performed $t$ tests to compare the SMR power at the C3 and C4 sites for the expert and novice groups. The SMR power for the expert group at the $\mathrm{C} 3$ and $\mathrm{C} 4$ sites was $1.357(\mathrm{SD}=0.545)$ and $1.346(\mathrm{SD}=0.661)$, respectively; the SMR power for the novice group at the $\mathrm{C} 3$ and C4 sites was 0.381 ( $S D=0.399)$ and $0.342(S D=0.356)$, respectively. We found that the expert and novice groups showed no significant difference in SMR power between the $\mathrm{C} 3$ and $\mathrm{C} 4$ sites (expert group: $(t(13)=1.598, p=.134)$; novice group: $(t(10)=1.578, p=.146))$.

\section{Discussion}

The aim of this study was to investigate the difference in SMR between experts and novices during a dart-throwing task. The results showed that the performance of dart throwing was preceded by a higher SMR power in the experts than in the novices, lending support for the hypothesis that lower sensorimotor activation, as reflected by higher SMR activity, is a characteristic of superior dart throwing.

The main finding of the present study was consistent with our hypothesis, in which we anticipated that SMR power in experts would be higher than in novices prior to dart release. Mann et al. (1996) suggested that SMR power was negatively correlated with the relay activity in the sensory motor cortex, which represented reduced conduction of somatosensory information during SMR activity. Subsequent studies showed that increasing SMR power was associated with reduced somatosensory and motor interference in an attention-demanding task (Egner \& Gruzelier, 2001, 2004). Furthermore, augmented SMR NFT was beneficial to declarative memory (Hoedlmoser et al., 2008), mental rotation (Doppelmayr \& Weber, 2011), novel music performance in children (Gruzelier, Foks, Steffert, Chen, \& Ros, 2014), and acting 
performance with a greater subjective flow state (Gruzelier et al., 2010). In addition, Ros et al. (2009) observed a significant reduction of simulated cataract surgical time due to the improvement in visuo-motor skills following augmented SMR training, which was accompanied by a reduction in anxiety. Collectively, these findings point to a relationship between augmented SMR activity and adaptive cognitive-attentional processing in attention-demanding tasks. The finding of higher SMR power in skilled participants suggests that their superior dart-throwing performance was preceded by a state of less somatosensory interference. In other words, skilled participants performing a highly coordinated dart throw may depend less on external somatosensory feedback to guide their movement. This adaptive cognitive-motor state results in a chunking execution of a well-practiced skill (Masters, 1992). That is, the dart throwers achieve more adaptive information processing of their motor skill during execution. This interpretation is in line with the characterization of an expert who can perform with high efficiency and refinement after a number of practice sessions (Kelly \& Garavan, 2005; Petersen et al., 1998), which can result in less attention investment during the actual performance (Bapi, Doya, \& Harner, 2000; Hikosaka, Nakamura, Sakai, \& Nakahara, 2002). This type of mental state is particularly helpful for performance during precision sports (Krane \& Williams, 2006).

A conceptualized automatic processing can be useful to explain the relationship between the SMR activity and adaptive mental states. An automatic process is parallel and effortless (Schneider \& Shiffrin, 1977). Neurophysiological studies have shown that the automatic pathway includes the bilateral ventral premotor region, the right middle frontal gyrus, the right caudate body, and lateral parts of the basal ganglia (Poldrack et al., 2005). Furthermore, evidence from a functional magnetic resonance imaging (fMRI) study showed that the weaker activity of the bilateral cerebellum, presupplementary motor area, cingulate cortex, left caudate nucleus, premotor cortex, parietal cortex, and prefrontal cortex were characteristics of the automatic process (Wu, Chan, \& Hallett, 2008). These findings suggest that the major motor network is involved in processing automatic movements, and reduced activity in the motor region during the execution movement could be related to an automatic process. This conceptualization is consistent with our findings, which showed that higher SMR power was observed in the preparation period of experts than that of novices. Therefore, less activation in the sensorimotor cortex may result in less interference with somatosensory information, which may be the major difference between an expert and a novice.

A generally higher $12-15 \mathrm{~Hz}$ power in experts than in novices suggests that skilled dart throwers perform superior dart throwing with lower interference in processing motor-related activity. The $12-15 \mathrm{~Hz}$ activity has been associated with the inhibition of motor activity (Mann et al., 1996) and has been shown to compose the dominant "standby" frequency of the integrated thalamocortical, somatosensory, and somatomotor pathways (Sterman \& Egner, 2006). Our findings suggest that experts performed the dartthrowing task with a globally reduced somatosensory process at the frontal, sensorimotor and parietal regions. This is consistent with previous studies demonstrating that better motor performance was closely related to lower activity among the premotor and primary motor areas (Babiloni et al., 2010; Del Percio et al., 2009) and replicates previous findings that suggest that skilled performers execute specialized tasks with lower cortical processing than novices (Meister et al., 2005). Moreover, the reduced 12-15 Hz power at the frontal region compared with other regions in novices suggests an effortful execution process that is closely related to the controlled process (Schneider \& Shiffrin, 1977).

The difference in skill level between dart throwers and novices is mainly reflected by the beta frequency band. Although alpha activity in the central area has been associated with sensory information processing over the sensorimotor area (Pfurtscheller \& Lopes Da Silva, 1999), this was not evidenced by this study. Moreover, we also failed to observe a difference in the theta frequency band between the two groups. However, the expert group exhibited a higher power with a frequency band of $15-18 \mathrm{~Hz}$ before dart throwing. The enhancement of $15-18 \mathrm{~Hz}$ EEG activity was related to increased cortical arousal and focus mechanisms affecting the sensorimotor cortex (Lubar \& Lubar, 1984). Previous findings showed that participants with augmented $15-18 \mathrm{~Hz}$ power at the sensorimotor area show reduced error rates (Egner \& Gruzelier, 2001). Similarly, participants exhibited a reduction of response time in attentional tasks and an increased P3b amplitude in oddball tasks with augmented SMR and $15-18 \mathrm{~Hz}$ power (Egner \& Gruzelier, 2004). These results suggest that the beta-related frequency band is closely related to adaptive attentional focus, which is restricted in the sensorimotor area. However, the best way to distinguish the effect of functional specificity in the adherent frequency band has been a controversial question (Gruzelier, 2014b). Our results could lend preliminary support to the legitimacy of using SMR power or a power of $15-18 \mathrm{~Hz}$ to examine the relationship between sensorimotor activation and fine motor performance (Gruzelier, 2014b).

Our control analysis on comparing SMR activity between the Resting and Dart Throwing conditions suggest that augmented SMR power is a specific EEG marker that exists in skilled dart throwers. We found that the expert group exhibited a significantly higher SMR power than the novice group in both the Dart Throwing and Resting conditions. SMR is categorized as an influential "standby" frequency, which reflects the activity of the integrated thalamocortical, somatosensory, and somatomotor pathways (Sterman \& Egner, 2006). Previous studies have revealed that SMR power can be blocked during the planning or simply the imagination of a motor act (Pfurtscheller \& Lopes Da Silva, 1999; Pfurtscheller \& Neuper, 1997). This finding suggests that the expert group performed both the dart throwing and resting tasks with less motor planning and imagination than the novice group. In addition, the expert group exhibited relatively higher SMR power in the Resting condition than in the Dart Throwing condition, whereas the novice group showed the opposite trend. One speculation regarding these results is that these two groups processed sensorimotor information during these two conditions differently because they perceived the context in a different way. Specifically, it is possible that the expert group maintained lower levels of information processing in the sensorimotor cortex during the dart-throwing task because they perceived the context in a comparably efficient way. On the contrary, the novice group performed the dart-throwing task with a relatively higher sensorimotor activation, which could be characteristic of inexperience and less integration of motor planning. This speculation is in line with the notion that both motor and visual experiences distinguish how participants proceed with the perception of actions between novices and experts. That is, experts perceive and recognize the specialized task more easily than novices (Hohmann, Troje, Olmos, \& Munzert, 2011). Taken together, these findings indicate that the augmented SMR power in expert dart throwers reflects a mental state with less motor planning and less attention-related sensorimotor processing within the two tasks than in novices. However, a more comparable control task with an event-locked segment would be a more advanced way to demonstrate the specificity of the task-specific EEG marker.

The evidence of different SMR power between the two groups can be inferred as a result of the variation of skill level instead of confounding variables before dart throwing, such as EOG and EMG activity. Our control analysis of EOG activity revealed that no difference was found in the fixation between the two groups before dart throwing. This evidence further suggests that the SMR variation between the two groups was due to the difference in sensorimotor information processing in the preparation period of dart throwing 
instead of the fixation. Moreover, we found that the expert group showed higher EMG power before dart throwing than the novice group. This finding is in line with the psychoneuromuscular theory (Driskell, Copper, \& Moran, 1994), which suggests that imagery generates neuromuscular responses similar to those of an actual experience. Specifically, practicing the execution of a movement mentally could lead to the transmission of the impulses to target muscles. This activation of muscular activity has been associated with the improvement of coordination patterns, which then results in the strengthening of motor programs in the motor cortex (Magill, 2001). Thus, the higher EMG power observed in the expert group relative to the novice group in this study could be the result of a large amount of practice, which leads to more integrated control of specific muscular activity. However, interpretations of the EMG results should be made with caution, as the dart-throwing task uses dynamic contraction, which is very sensitive to the morphological properties of the muscle (Farina, Merletti, \& Enoka, 2004; Farina, 2006). Therefore, further investigation of the relationship between EMG activity and SMR power before dart throwing is required.

Caution should be exercised when interpreting this study's findings due to several limitations. Although the antecedent role of SMR provides some support for its causal role, future interventional studies to ascertain this relationship are warranted. The functional meaning of SMR requires further investigation by comparing the best and worst performance within highly skilled performers. Although some studies have found that higher SMR power was related to superior attentional performance (Egner \& Gruzelier, 2001, 2004; Hoedlmoser et al., 2008), others have indicated that SMR was not essential in musical performance (Egner \& Gruzelier, 2003) or for an audiovisual Go/NoGo cursor movement task (Boulay, Sarnacki, Wolpaw, \& McFarland, 2011). Additionally, EEG provides real-time cortical processing information during motor performance (Divekar \& John, 2013), but it is limited in its ability to generate information on how the sensorimotor cortex works with the other relevant regions during motor execution (Thompson, Steffert, Ros, Leach, \& Gruzelier, 2008). The functional role of SMR requires more specification, and neuroimaging tools, such as fMRI, can be incorporated to generate a more complete understanding of the function of the sensorimotor cortex. Moreover, in a neurofeedback training study, SMR power and subjective measures, such as Flow State Scales (Jackson \& Eklund, 2004), were recommended for future studies. Previous research has demonstrated the benefit of neurofeedback in the sports domain (Arns, Kleinnijenhuis, Fallahpour, \& Breteler, 2008; Gruzelier et al., 2010; Hammond, 2007; Kao, Huang, \& Hung, 2014; Landers et al., 1991). More neurofeedback training designs are not only useful to examine the causal relationship between SMR activity and motor performance but may also shed light on the practical implications for the regulation of SMR. Furthermore, the electrooculography detected in this study should be examined with more refined tools, although we found that no significant difference was observed for the fixation reflected by the EOG data two seconds before dart throwing. This observation may be refined by using more sophisticated electrooculographic measurements, such as eye trackers. Finally, the limited time period of EEG power analysis needs to be extended further to elucidate the fluctuation of EEG power in more detail. We examined the variation of EEG power using only twosecond time windows prior to dart throwing. We recommend that future studies explore the time window during the entire skill execution process to further our understanding of the mental process associated with motor skill performance.

In conclusion, as supported by the EEG spectral analysis of SMR in this study, dart-throwing expertise is reflected by a higher SMR power prior to dart release. This finding implies that experts rely less on somatosensory information processing to guide their throwing movement in a relatively more adaptive manner. On the contrary, novices tend to use feedback from kinesthetic information, as reflected by a lower SMR power, to perform the throwing task. These findings advance our understanding of an adaptive mental state in skilled motor performance and also shed light on the potential of regulating SMR activity to improve attention during the performance of sports.

\section{Acknowledgment}

Tsung-Min Hung's work was supported in part by the Ministry of Science and Technology (Taiwan) under grant NSC93-2413-H154-010.

\section{References}

Arns, M., Kleinnijenhuis, M., Fallahpour, K., \& Breteler, R. (2008). Golf performance enhancement and real-life neurofeedback training using personalized event-locked EEG profiles. Journal of Neurotherapy, 11(4), 11-18. http://dx.doi. org/10.1080/10874200802149656

Babiloni, C., Del Percio, C., Iacoboni, M., Infarinato, F., Lizio, R., Marzano, N., \& Eusebi, F. (2008). Golf putt outcomes are predicted by sensorimotor cerebral EEG rhythms. The Journal of Physiology, 586(1), 131-139. http://dx.doi.org/10. 1113/jphysiol.2007.141630

Babiloni, C., Marzano, N., Infarinato, F., Iacoboni, M., Rizza, G., Aschieri, P., \& Del Percio, C. (2010). Neural efficiency of experts' brain during judgment of actions: a high-resolution EEG study in elite and amateur karate athletes. Behavioural Brain Research, 207(2), 466-475. http://dx.doi.org/10.1016/j.bbr.2009.10.034

Bapi, R. S., Doya, K., \& Harner, A. M. (2000). Evidence for effector independent and dependent representations and their differential time course of acquisition during motor sequence learning. Experimental Brain Research, 132(2), 149-162. http://dx.doi.org/10.1007/s002219900332

Boulay, C. B., Sarnacki, W. A., Wolpaw, J. R., \& McFarland, D. J. (2011). Trained modulation of sensorimotor rhythms can affect reaction time. Clinical Neurophysiology, 122(9), 1820-1826. http://dx.doi.org/10.1016/j.clinph.2011. 02.016

Dayan, E., \& Cohen, L. G. (2011). Neuroplasticity subserving motor skill learning. Neuron, 72(3), 443-454. http://dx.doi.org/10.1016/j.neuron.2011.10.008

Del Percio, C., Babiloni, C., Marzano, N., Iacoboni, M., Infarinato, F., Vecchio, F., \& Eusebi, F. (2009). Neural efficiency of athletes' brain for upright standing: a high-resolution EEG study. Brain Research Bulletin, 79(3-4), 193-200. http://dx. doi.org/10.1016/j.brainresbull.2009.02.001

Divekar, N. V., \& John, L. R. (2013). Neurophysiological, behavioural and perceptual differences between wrist flexion and extension related to sensorimotor monitoring as shown by corticomuscular coherence. Clinical Neurophysiology, 124(1), 136-147. http://dx.doi.org/10.1016/j.clinph.2012.07.019

Doppelmayr, M., \& Weber, E. (2011). Effects of SMR and theta/beta neurofeedback on reaction times, spatial abilities, and creativity. Journal of Neurotherapy, 15(2), 115-129. http://dx.doi.org/10.1080/10874208.2011.570689

Driskell, J. E., Copper, C., \& Moran, A. (1994). Does mental practice enhance performance? Journal of Applied Psychology, 79(4), 481-492. http://dx.doi.org/ 10.1037/0021-9010.79.4.481

Egner, T., \& Gruzelier, J. H. (2001). Learned self-regulation of EEG frequency components affects attention and event-related brain potentials in humans. Neuroreport, 12(18), 4155-4159. http://dx.doi.org/10.1097/00001756200112210-00058

Egner, T., \& Gruzelier, J. H. (2003). Ecological validity of neurofeedback: modulation of slow wave EEG enhances musical performance. Neuroreport, 14(9), 1221-1224. http://dx.doi.org/10.1097/01.wnr.0000081875.45938.d1

Egner, T., \& Gruzelier, J. H. (2004). EEG biofeedback of low beta band components: frequency-specific effects on variables of attention and event-related brain potentials. Clinical Neurophysiology, 115(1), 131-139. http://dx.doi.org/10. 1016/S1388-2457(03)353-5

Farina, D. (2006). Interpretation of the surface electromyogram in dynamic contractions. Exercise and Sport Sciences Reviews, 34(3), 121-127. http://dx.doi. org/10.1249/00003677-200607000-00006

Farina, D., Merletti, R., \& Enoka, R. M. (2004). The extraction of neural strategies from the surface EMG. Journal of Applied Physiology (Bethesda, Md.: 1985), 96(4), 1486-1495. http://dx.doi.org/10.1152/japplphysiol.01070.2003

Fogassi, L., \& Luppino, G. (2005). Motor functions of the parietal lobe. Current Opinion in Neurobiology, 15(6), 626-631. http://dx.doi.org/10.1016/j.conb. 2005.10.015

Gruzelier, J., Egner, T., \& Vernon, D. (2006). Chapter 27 validating the efficacy of neurofeedback for optimising performance. Progress in Brain Research, 159, 421-431. http://dx.doi.org/10.1016/S0079-6123(06)59027-2

Gruzelier, J. H. (2014a). Differential effects on mood of 12-15 (SMR) and 15-18 (beta1) Hz neurofeedback. International Journal of Psychophysiology, 93(1), 112-115. http://dx.doi.org/10.1016/j.ijpsycho.2012.11.007

Gruzelier, J. H. (2014b). EEG-neurofeedback for optimising performance. I: a review of cognitive and affective outcome in healthy participants. Neuroscience and Biobehavioral Reviews, 44, 124-141. http://dx.doi.org/10.1016/j.neubiorev. 2013.09.015 
Gruzelier, J. H., Foks, M., Steffert, T., Chen, M. J. L., \& Ros, T. (2014). Beneficial outcome from EEG-neurofeedback on creative music performance, attention and well-being in school children. Biological Psychology, 95(1), 86-95. http:// dx.doi.org/10.1016/j.biopsycho.2013.04.005

Gruzelier, J., Inoue, A., Smart, R., Steed, A., \& Steffert, T. (2010). Acting performance and flow state enhanced with sensory-motor rhythm neurofeedback comparing ecologically valid immersive VR and training screen scenarios. Neuroscience Letters, 480(2), 112-116. http://dx.doi.org/10.1016/j.neulet.2010. 06.019

Hammond, D. (2007). Neurofeedback for the enhancement of athletic performance and physical balance. The Journal of the American Board of Sport Psychology, 1, 1-9. Retrieved from: http://www.mendeley.com/research/neurofeedbackenhancement-athletic-performance-physical-balance/

Hikosaka, O., Nakamura, K., Sakai, K. \& Nakahara, H. (2002). Central mechanisms of motor skill learning. Current Opinion in Neurobiology, 12(2), 217-222. http://dx. doi.org/10.1016/S0959-4388(02)00307-0

Hoedlmoser, K., Pecherstorfer, T., Gruber, G., Anderer, P., Doppelmayr, M., Klimesch, W., \& Schabus, M. (2008). Instrumental conditioning of human sensorimotor rhythm $(12-15 \mathrm{~Hz})$ and its impact on sleep as well as declarative learning. Sleep, 31(10), 1401-1408. Retrieved from: http://www. pubmedcentral.nih.gov/articlerender. fcgi? artid $=2572745 \&$ tool $=$ pmcentrez\&rendertype=Abstract

Hohmann, T., Troje, N. F., Olmos, A., \& Munzert, J. (2011). The influence of motor expertise and motor experience on action and actor recognition. Journal of Cognitive Psychology, 23(4), 403-415. http://dx.doi.org/10.1080/20445911. 2011.525504

Howe, R. C., \& Sterman, M. B. (1973). Somatosensory system evoked potentials during waking behavior and sleep in the cat. Electroencephalography and Clinical Neurophysiology, 34(6), 605-618. http://dx.doi.org/10.1016/00134694(73)90006-0

Jackson, S., Eklund, R. C., The flow scales manual, 2004, Morgantown, WV: Fitness Information Technology.

Jasper, H. H. (1958). Report of the committee on methods of clinical examination in electroencephalography. Electroencephalography and Clinical Neurophysiology, 10(2), 370-375. http://dx.doi.org/10.1016/0013-4694(58)90053-1

Kao, S., Huang, C., \& Hung, T. (2014). Neurofeedback training reduces frontal midline theta and improves putting performance in expert golfers. Journal of Applied Sport Psychology, 26(3), 271-286. http://dx.doi.org/10.1080/10413200 2013.855682

Kelly, A. M. C., \& Garavan, H. (2005). Human functional neuroimaging of brain changes associated with practice. Cerebral Cortex, 15(8), 1089-1102. http://dx doi.org/10.1093/cercor/bhi005

Krane, V., \& Williams, J. M. (2006). Psychological characteristics of peak performance. In J. M. Williams (Ed.), Applied sport psychology: personal growth to peak performance (5th ed., pp. 158-170). New York, NY: McGraw-Hill.

Landers, D. M., Petruzzello, S. J., Salazar, W., Crews, D. J., Kubitz, K. A., Gannon, T. L., \& Han, M. (1991). The influence of electro cortical biofeedback on performance in pre-elite archers. Medicine and Science in Sports and Exercise, 23(1), 123-129. http://dx.doi.org/10.1249/00005768-199101000-00018

Lubar, J. O., \& Lubar, J. F. (1984). Electroencephalographic biofeedback of SMR and beta for treatment of attention deficit disorders in a clinical setting. Biofeedback and Self-Regulation, 9(1), 1-23. http://dx.doi.org/10.1007/BF00998842

Magill, R.A. (2001). Motor Learning: Concepts and Applications (Vol. 8). Retrieved from: http://books.google.de/books/about/Motor_Learning. html?id=TMzHv28M9zAC\&pgis=1

Mann, C. A., Sterman, M. B., \& Kaiser, D. A. (1996). Suppression of EEG rhythmic frequencies during somato-motor and visuo-motor behavior. International Journal of Psychophysiology, 23(1-2), 1-7. http://dx.doi.org/10.1016/01678760(96)36-0
Manor, B. R., \& Gordon, E. (2003). Defining the temporal threshold for ocular fixation in free-viewing visuocognitive tasks. Journal of Neuroscience Methods, 128(1-2), 85-93. http://dx.doi.org/10.1016/S0165-0270(03)151-1

Masters, R. S. W. (1992). Knowledge, knerves and know-how: the role of explicit versus implicit knowledge in the breakdown of a complex motor skill under pressure. British Journal of Psychology, 83(3), 343-358. http://dx.doi.org/10. 1111/j.2044-8295.1992.tb02446.x

Meister, I., Krings, T., Foltys, H., Boroojerdi, B., Müller, M., Töpper, R., \& Thron, A. (2005). Effects of long-term practice and task complexity in musicians and nonmusicians performing simple and complex motor tasks: Implications for cortical motor organization. Human Brain Mapping, 25(3), 345-352. http://dx doi.org/10.1002/hbm.20112

Milton, J., Solodkin, A., Hluštík, P., \& Small, S. L. (2007). The mind of expert motor performance is cool and focused. Neuroimage, 35(2), 804-813. http://dx.doi. org/10.1016/j.neuroimage.2007.01.003

Niemarkt, H. J., Jennekens, W., Pasman, J. W., Katgert, T., Van Pul, C., Gavilanes, A. W. D., \& Andriessen, P. (2011). Maturational changes in automated EEG spectral power analysis in preterm infants. Pediatric Research, 70(5), 529-534. http://dx.doi.org/10.1203/PDR.0b013e31822d748b

Petersen, S. E., van Mier, H., Fiez, J. A., \& Raichle, M. E. (1998). The effects of practice on the functional anatomy of task performance. Proceedings of the National Academy of Sciences of the United States of America, 95(3), 853-860. http://dx. doi.org/10.1073/pnas.95.3.853

Pfurtscheller, G., \& Da Silva, Lopes. (1999). Event-related EEG/MEG synchronization and desynchronization. Basic Principles Clinical Neurophysiology, 110(11), 1842-1857. http://dx.doi.org/10.1016/S1388-2457(99)141-8

Pfurtscheller, G., \& Neuper, C. (1997). Motor imagery activates primary sensorimotor area in humans. Neuroscience Letters, 239(2-3), 65-68. http://dx. doi.org/10.1016/S0304-3940(97)889-6

Poldrack, R. A., Sabb, F. W., Foerde, K., Tom, S. M., Asarnow, R. F., Bookheimer, S. Y., \& Knowlton, B. J. (2005). The neural correlates of motor skill automaticity. The Journal of neuroscience: the official journal of the Society for Neuroscience, 25(22) 5356-5364. http://dx.doi.org/10.1523/JNEUROSCI.3880-04.2005

Ros, T., Moseley, M. J., Bloom, P. A., Benjamin, L., Parkinson, L. A., \& Gruzelier, J. H. (2009). Optimizing microsurgical skills with EEG neurofeedback. BMC Neuroscience, 10, 87. http://dx.doi.org/10.1186/1471-2202-10-87

Schneider, W., \& Shiffrin, R. M. (1977). Controlled and automatic human information processing: I. Detection, search, and attention. Psychological Review, 84, 1. http://dx.doi.org/10.1037/0033-295X

Semlitsch, H. V., Anderer, P., Schuster, P., \& Presslich, O. (1986). A solution for reliable and valid reduction of ocular artifacts, applied to the P300 ERP. Psychophysiology, 23(6), 695-703. http://dx.doi.org/10.1111/j 1469-8986.1986. tb00696.x

Sterman, M. B., \& Egner, T. (2006). Foundation and practice of neurofeedback for the treatment of epilepsy. Applied Psychophysiology Biofeedback, 31(1), 21-35. http://dx.doi.org/10.1007/s10484-006-9002-x

Thompson, T., Steffert, T., Ros, T., Leach, J., \& Gruzelier, J. (2008). EEG applications for sport and performance. Methods, 45(4), 279-288. http://dx.doi.org/10. 1016/j.ymeth.2008.07.006

Vance, J., Wulf, G., Töllner, T., McNevin, N., \& Mercer, J. (2004). EMG activity as a function of the performer's focus of attention. Journal of Motor Behavior, 36(4), 450-459. http://dx.doi.org/10.3200/JMBR.36.4450-459

Vernon, D., Egner, T., Cooper, N., Compton, T., Neilands, C., Sheri, A., \& Gruzelier, J. (2003). The effect of training distinct neurofeedback protocols on aspects of cognitive performance. International Journal of Psychophysiology, 47(1), 75-85. http://dx.doi.org/10.1016/S0167-8760(02)91-0

Wu, T., Chan, P., \& Hallett, M. (2008). Modifications of the interactions in the motor networks when a movement becomes automatic. The Journal of Physiology, 586, 4295-4304. http://dx.doi.org/10.1113/jphysiol.2008.153445 